\title{
Some Properties for Certain Class of Analytic Functions with Varying Arguments
}

\author{
M. K. Aouf, A. O. Mostafa, A. Shamandy, and E. A. Adwan \\ Department of Mathematics, Faculty of Science, Mansoura University, Mansoura 35516, Egypt \\ Correspondence should be addressed to E. A. Adwan; eman.a2009@yahoo.com \\ Received 21 December 2012; Accepted 11 March 2013 \\ Academic Editor: Tohru Ozawa
}

Copyright ( 2013 M. K. Aouf et al. This is an open access article distributed under the Creative Commons Attribution License, which permits unrestricted use, distribution, and reproduction in any medium, provided the original work is properly cited.

We introduce a new class $V(k, \lambda, A, B)$ of analytic functions with varying arguments in the open unit disc $U=\{z \in \mathbb{C}:|z|<1\}$ defined by the Salagean operator. The object of the present paper is to determine coefficient estimates, extreme points, and distortion theorems for functions $f(z)$ belonging to the class $V(k, \lambda, A, B)$.

\section{Introduction}

Let $\mathscr{A}$ denote the class of functions of the form

$$
f(z)=z+\sum_{n=2}^{\infty} a_{n} z^{n}
$$

which are analytic in the open unit disc $U=\{z \in \mathbb{C}:$ $|z|<1\}$. If $f$ and $g$ are analytic functions in $U$, we say that $f$ is subordinate to $g$, written $f \prec g$ if there exists a Schwarz function $w$, which (by definition) is analytic in $U$ with $w(0)=0$ and $|w(z)|<1$ for all $z \in U$, such that $f(z)=g(w(z)), z \in U$. Furthermore, if the function $g$ is univalent in $U$, then we have the following equivalence (cf, e.g., $[1,2])$ :

$$
f(z) \prec g(z) \Longleftrightarrow f(0)=g(0), f(U) \subset g(U) .
$$

For function $f(z) \in \mathscr{A}$, Salagean [3] defined the differential operator $D^{k}$ as follows:

$$
\begin{gathered}
D^{0} f(z)=f(z), \\
D^{1} f(z)=D f(z)=z f^{\prime}(z), \\
D^{k} f(z)=D\left(D^{k-1} f(z)\right), \\
\left(k \in \mathbb{N}_{0}=\mathbb{N} \cup\{0\}, \mathbb{N}=\{1,2, \ldots\}\right) .
\end{gathered}
$$

For $\lambda \geq 0,-1 \leq A<B \leq 1,0<B \leq 1, k \in \mathbb{N}_{0}$, and for all $z \in U$, let $S(k, \lambda, A, B)$ denotes the subclass of $\mathscr{A}$ consisting of functions $f(z)$ of the form (1) and satisfying the analytic criterion:

$$
(1-\lambda) \frac{D^{k} f(z)}{z}+\lambda\left(D^{k} f(z)\right)^{\prime} \prec \frac{1+A z}{1+B z} .
$$

It is noticed that for suitable choices of $k, \lambda, A$, and $B$, we obtain the following subclasses studied by various authors:

(1) $S(k, \lambda, A, B)=G_{k}(\lambda, 1, A, B)=G_{k}(\lambda, A, B)(\lambda \geq$ $0,-1 \leq A<B \leq 1,0<B \leq 1, k \in \mathbb{N}_{0}$ ) (see Sivasubramanian et al. [4], with $b=1$ );

(2) $S(k, \lambda, 2 \alpha-1,1)=S \Im(k, \lambda, \alpha)\left(k \in \mathbb{N}_{0}, 0 \leq \alpha<\right.$ $1, \lambda \geq 0)$ (see Aouf et al. [5], with $b=1$ );

(3) $S(0, \lambda, 2 \alpha-1,1)=B(\lambda, \alpha)(0 \leq \alpha<1,0 \leq \lambda \leq 1)$ (see Chunyi and Owa [6]);

(4) $S(0,0,2 \alpha-1,1)=B(\alpha)(0 \leq \alpha<1)($ see Chen $[7,8]$ and Goel [9]);

(5) $S(1,0,2 \alpha-1,1)=C(\alpha)(0 \leq \alpha<1)$ (see Srivastava and Owa [10]).

Also we note the following.

(1) Putting $k=0$ in (4), the class $S(0, \lambda, A, B)$ reduces to the class $S(\lambda, A, B)=\left\{(1-\lambda)(f(z) / z)+\lambda f^{\prime}(z) \prec((1+\right.$ $A z) /(1+B z)), \lambda \geq 0,-1 \leq A<B \leq 1,0<B \leq 1\}$. 
Definition 1 (see [11]). A function $f(z)$ defined by (1) is said to be in the class $V\left(\theta_{n}\right)$ if $f(z) \in \mathscr{A}$ and $\arg \left(a_{n}\right)=\theta_{n}$ for all $n \geq 2$. If, furthermore, there exists a real number $\beta$ such that

$$
\theta_{n}+(n-1) \beta \equiv \pi(\bmod 2 \pi),
$$

then $f(z)$ is said to be in the class $V\left(\theta_{n} ; \beta\right)$. The union of $V\left(\theta_{n} ; \beta\right)$ taken over all possible sequences $\left\{\theta_{n}\right\}$ and all possible real numbers $\beta$ is denoted by $V$.

Let $V(k, \lambda, A, B)$ denote the subclass of $V$ consisting of functions $f(z)$ in $S(k, \lambda, A, B)$.

We note the following:

(1) $V(0, \lambda, 2 \alpha-1,1)=V_{\lambda}(\alpha)(0 \leq \alpha<1, \lambda \geq 0)$ (see Aouf et al. [12]);

(2) $V(0,0,2 \alpha-1,1)=B_{\alpha}(0 \leq \alpha<1)$ (see Srivastava and Owa [10]);

(3) $V(1,0,2 \alpha-1,1)=C_{\alpha}(0 \leq \alpha<1)$ (see Srivastava and Owa [10]).

Also we note that

(i) $V(k, \lambda, 2 \alpha-1,1)=V \mathfrak{I}(k, \lambda, \alpha)\left(k \in \mathbb{N}_{0}, 0 \leq \alpha<\right.$ $1, \lambda \geq 0$ ) denotes the subclass of $V$, consisting of functions $f(z)$ belonging to the class $S \Im(k, \lambda, \alpha)$;

(ii) $V(0, \lambda, A, B)=V(\lambda, A, B)(\lambda \geq 0,-1 \leq A<B \leq$ $1,0<B \leq 1)$ denotes the subclass of $V$, consisting of functions $f(z)$ belonging to the class $S(\lambda, A, B)$.

\section{Coefficient Estimates}

Unless otherwise mentioned, we will assume in the reminder of this paper that $\lambda \geq 0,-1 \leq A<B \leq 1,0<B \leq 1$ and $k \in \mathbb{N}_{0}$.

Theorem 2. Let the function $f(z) \in \mathscr{A}$ be of the form (1), if

$$
\sum_{n=2}^{\infty} n^{k}[1+\lambda(n-1)](1+B)\left|a_{n}\right| \leq(B-A),
$$

then $f(z) \in S(k, \lambda, A, B)$.

Proof. A function $f(z)$ of the form (1) belongs to the class $S(k, \lambda, A, B)$ if and only if there exists a function $w,|w(z)| \leq$ $|z|$, such that

$$
(1-\lambda) \frac{D^{k} f(z)}{z}+\lambda\left(D^{k} f(z)\right)^{\prime}=\frac{1+A w(z)}{1+B w(z)},
$$

or, equivalently,

$$
\left|\frac{(1-\lambda)\left(D^{k} f(z) / z\right)+\lambda\left(D^{k} f(z)\right)^{\prime}-1}{B\left[(1-\lambda)\left(D^{k} f(z) / z\right)+\lambda\left(D^{k} f(z)\right)^{\prime}\right]-A}\right|<1 .
$$

Thus, it is sufficient to prove that

$$
\begin{aligned}
& \left|(1-\lambda) \frac{D^{k} f(z)}{z}+\lambda\left(D^{k} f(z)\right)^{\prime}-1\right| \\
& \quad-\left|B\left[(1-\lambda) \frac{D^{k} f(z)}{z}+\lambda\left(D^{k} f(z)\right)^{\prime}\right]-A\right|<0 .
\end{aligned}
$$

Indeed, letting $|z|=r(0 \leq r<1)$ we have

$$
\begin{aligned}
\mid(1- & \lambda) \frac{D^{k} f(z)}{z}+\lambda\left(D^{k} f(z)\right)^{\prime}-1 \mid \\
& -\left|B\left[(1-\lambda) \frac{D^{k} f(z)}{z}+\lambda\left(D^{k} f(z)\right)^{\prime}\right]-A\right| \\
= & \left|\sum_{n=2}^{\infty} n^{k}[1+\lambda(n-1)] a_{n} z^{n-1}\right| \\
& -\left|(B-A)+\sum_{n=2}^{\infty} B n^{k}[1+\lambda(n-1)] a_{n} z^{n-1}\right| \\
\leq & \left(\sum_{n=2}^{\infty} n^{k}[1+\lambda(n-1)]\left|a_{n}\right| r^{n-1}\right. \\
& \left.-(B-A)+\sum_{n=2}^{\infty} B n^{k}[1+\lambda(n-1)]\left|a_{n}\right| r^{n-1}\right) \\
< & \sum_{n=2}^{\infty} n^{k}[1+\lambda(n-1)](1+B)\left|a_{n}\right|-(B-A) .
\end{aligned}
$$

In view of (6), the last inequality is less than zero; hence $f(z) \in S(k, \lambda, A, B)$. This completes the proof of Theorem 2 .

Theorem 3. Let the function $f(z) \in \mathscr{A}$ be of the form (1), then $f(z)$ is in the class $V(k, \lambda, A, B)$ if and only if

$$
\sum_{n=2}^{\infty} n^{k}[1+\lambda(n-1)](1+B)\left|a_{n}\right| \leq(B-A) .
$$

Proof. In view of Theorem 2, we need only to show that each function $f(z)$ from the class $V(k, \lambda, A, B)$ satisfies the coefficient inequality (6). Let $f(z) \in V(k, \lambda, A, B)$. Then, by (8) and (1), we have

$$
\left|\frac{\sum_{n=2}^{\infty} n^{k}[1+\lambda(n-1)] a_{n} z^{n-1}}{(B-A)+\sum_{n=2}^{\infty} B n^{k}[1+\lambda(n-1)] a_{n} z^{n-1}}\right|<1 .
$$

Since $f(z) \in V, f(z)$ lies in the class $V\left(\theta_{n}, \beta\right)$ for some sequence $\left\{\theta_{n}\right\}$ and a real number $\beta$ such that $\theta_{n}+(n-1) \beta \equiv$ $\pi(\bmod 2 \pi)(n \geq 2)$, set $z=r e^{i \beta}$ in the above inequality and since $\operatorname{Re}\{w(z)\}<|w(z)|<1$, then

$$
\frac{\sum_{n=2}^{\infty} n^{k}[1+\lambda(n-1)]\left|a_{n}\right| r^{n-1}}{(B-A)-\sum_{n=2}^{\infty} B n^{k}[1+\lambda(n-1)]\left|a_{n}\right| r^{n-1}}<1 .
$$

It is clear that the denominator of the left-hand said cannot vanish for $r \in[0,1)$. Moreover, it is positive for $r=0$ and in consequence for $r \in[0,1)$. Thus, we have

$$
\sum_{n=2}^{\infty} n^{k}[1+\lambda(n-1)](1+B)\left|a_{n}\right| r^{n-1} \leq(B-A),
$$

which, upon letting $r \rightarrow 1^{-}$, readily yields the assertion (11). This completes the proof of Theorem 3. 
Corollary 4. Let the function $f(z) \in \mathscr{A}$ defined by (1) be in the class $V(k, \lambda, A, B)$, then

$$
\left|a_{n}\right| \leq \frac{(B-A)}{n^{k}[1+\lambda(n-1)](1+B)} \quad(n \geq 2) .
$$

The result is sharp for the function

$$
f(z)=z+\frac{(B-A)}{n^{k}[1+\lambda(n-1)](1+B)} e^{i \theta_{n}} z^{n} \quad(n \geq 2) .
$$

\section{Distortion Theorems}

Theorem 5. Let the function $f(z) \in \mathscr{A}$ defined by (1) be in the class $V(k, \lambda, A, B)$. Then

$$
\begin{aligned}
|z|- & \frac{(B-A)}{2^{k}(1+B)(1+\lambda)}|z|^{2} \\
& \leq|f(z)| \leq|z|+\frac{(B-A)}{2^{k}(1+B)(1+\lambda)}|z|^{2} .
\end{aligned}
$$

The result is sharp.

Proof. Since

$$
\Psi(n)=n^{k}[1+\lambda(n-1)](1+B)
$$

is an increasing function of $n(n \geq 2)$, from Theorem 2 , we have

$$
\begin{aligned}
& 2^{k}(1+B)(1+\lambda) \sum_{n=2}^{\infty}\left|a_{n}\right| \\
& \quad \leq \sum_{n=2}^{\infty} n^{k}[1+\lambda(n-1)](1+B)\left|a_{n}\right| \leq(B-A),
\end{aligned}
$$

that is,

$$
\sum_{n=2}^{\infty}\left|a_{n}\right| \leq \frac{(B-A)}{2^{k}(1+B)(1+\lambda)}
$$

Thus,

$$
\begin{aligned}
|f(z)| & =\left|z+\sum_{n=2}^{\infty} a_{n} z^{n}\right| \leq|z|+\sum_{n=2}^{\infty}\left|a_{n}\right||z|^{2} \\
& \leq|z|+\frac{(B-A)}{2^{k}(1+B)(1+\lambda)}|z|^{2} .
\end{aligned}
$$

Similarly, we get

$$
\begin{aligned}
|f(z)| & \geq|z|-\sum_{n=2}^{\infty}\left|a_{n}\right|\left|z^{n}\right| \geq|z|-\sum_{n=2}^{\infty}\left|a_{n}\right||z|^{2} \\
& \geq|z|-\frac{(B-A)}{2^{k}(1+B)(1+\lambda)}|z|^{2} .
\end{aligned}
$$

Finally, the result is sharp for the function

$$
f(z)=z+\frac{(B-A)}{2^{k}(1+B)(1+\lambda)} e^{i \theta_{2}} z^{2}
$$

at $z= \pm|z| e^{-i \theta_{2}}$

This completes the proof of Theorem 5 .
Corollary 6. Under the hypotheses of Theorem 5, $f(z)$ is included in a disc with center at the origin and radius $r_{1}$ given by

$$
r_{1}=1+\frac{(B-A)}{2^{k}(1+B)(1+\lambda)} .
$$

Theorem 7. Let the function $f(z) \in \mathscr{A}$ defined by (1) belong to the class $V(k, \lambda, A, B)(k \geq 1)$. Then

$$
\begin{aligned}
& 1-\frac{(B-A)}{2^{k-1}(1+B)(1+\lambda)}|z| \\
& \quad \leq\left|f^{\prime}(z)\right| \leq 1+\frac{(B-A)}{2^{k-1}(1+B)(1+\lambda)}|z| .
\end{aligned}
$$

The result is sharp for the function $f(z)$ given by (23) at $z=$ $\pm|z| e^{-i \theta_{2}}$.

Proof. Since $\{n \Psi(n)\}$, where $\Psi(n)$ given by (18) is increasing function of $n(n \geq 2)$, in view of Theorem 3, we have

$$
\begin{aligned}
& \frac{2^{k}(1+B)(1+\lambda)}{2} \sum_{n=2}^{\infty} n\left|a_{n}\right| \\
& \quad \leq \sum_{n=2}^{\infty} n^{k}[1+\lambda(n-1)](1+B)\left|a_{n}\right| \leq(B-A),
\end{aligned}
$$

that is,

$$
\sum_{n=2}^{\infty} n\left|a_{n}\right| \leq \frac{(B-A)}{2^{k-1}(1+B)(1+\lambda)} .
$$

Thus,

$$
\begin{aligned}
\left|f^{\prime}(z)\right| & =\left|1+\sum_{n=2}^{\infty} n a_{n} z^{n-1}\right| \leq 1+\sum_{n=2}^{\infty} n\left|a_{n}\right||z| \\
& \leq 1+\frac{(B-A)}{2^{k-1}(1+B)(1+\lambda)}|z| .
\end{aligned}
$$

Similarly, we get

$$
\begin{aligned}
\left|f^{\prime}(z)\right| & \geq 1-\sum_{n=2}^{\infty} n\left|a_{n}\right|\left|z^{n-1}\right| \geq 1-\sum_{n=2}^{\infty} n\left|a_{n}\right||z| \\
& \geq 1-\frac{(B-A)}{2^{k-1}(1+B)(1+\lambda)}|z| .
\end{aligned}
$$

Finally, the result is sharp for the function $f(z)$ given by (23). This completes the proof of Theorem 7 .

Corollary 8. Let the function $f(z) \in \mathscr{A}$ defined by (1) be in the class $V(k, \lambda, A, B)$. Then $f^{\prime}(z)$ is included in a disc with center at the origin and radius $r_{2}$ given by

$$
r_{2}=1+\frac{(B-A)}{2^{k-1}(1+B)(1+\lambda)} .
$$




\section{Extreme Points}

Theorem 9. Let the function $f(z) \in \mathscr{A}$ defined by (1) be in the class $V(k, \lambda, A, B)$, with $\arg \left(a_{n}\right)=\theta_{n}$ where $\left[\theta_{n}+(n-1) \beta\right] \equiv$ $\pi(\bmod 2 \pi)$. Define $f_{1}(z)=z$ and

$$
f_{n}(z)=z+\frac{(B-A)}{n^{k}[1+\lambda(n-1)](1+B)} e^{i \theta_{n}} z^{n} .
$$

Then $f(z)$ is in the class $V(k, \lambda, A, B)$ if and only if it can be expressed in the form

$$
f(z)=\sum_{n=1}^{\infty} \mu_{n} f_{n}(z)
$$

where $\mu_{n} \geq 0(n \geq 1)$ and $\sum_{n=1}^{\infty} \mu_{n}=1$.

Proof. If $f(z)=\sum_{n=1}^{\infty} \mu_{n} f_{n}(z)$ with $\sum_{n=1}^{\infty} \mu_{n}=1$ and $\mu_{n} \geq 0$, then

$$
\begin{gathered}
\sum_{n=2}^{\infty} n^{k}[1+\lambda(n-1)](1+B) \frac{(B-A)}{n^{k}[1+\lambda(n-1)](1+B)} \mu_{n} \\
=\sum_{n=2}^{\infty}(B-A) \mu_{n}=(B-A)\left(1-\mu_{1}\right) \leq(B-A) .
\end{gathered}
$$

Hence, $f(z) \in V(k, \lambda, A, B)$.

Conversely, let the function $f(z)$ defined by (1) belong to the class $V(k, \lambda, A, B)$, and define

$$
\begin{gathered}
\mu_{n}=\frac{n^{k}[1+\lambda(n-1)](1+B)}{(B-A)}\left|a_{n}\right|, \\
\mu_{1}=1-\sum_{n=2}^{\infty} \mu_{n} .
\end{gathered}
$$

From Theorem $3, \sum_{n=1}^{\infty} \mu_{n} \leq 1$ and so $\mu_{n} \geq 0$. Since $\mu_{n} f_{n}(z)=$ $\mu_{n} z+a_{n} z^{n}$, then

$$
\sum_{n=1}^{\infty} \mu_{n} f_{n}(z)=z+\sum_{n=2}^{\infty} a_{n} z^{n}=f(z) .
$$

This completes the proof of Theorem 9.

Remark 10. Specializing $k, \lambda, A$, and $B$ in the above results, we obtain the corresponding results for the corresponding classes $V \mathfrak{I}(k, \lambda, \alpha)$ and $V(\lambda, A, B)$ defined in Section 1 .

\section{Acknowledgments}

The authors would like to thank the referees of the paper for their helpful suggestions.

\section{References}

[1] T. Bulboaca, Differential Subordinations and Superordinations, Recent Results, House of Scientific Book, Cluj-Napoca, Romania, 2005.
[2] S. S. Miller and P. T. Mocanu, Differential Subordinations: Theory and Applications, vol. 225 of Monographs and Textbooks in Pure and Applied Mathematics, Marcel Dekker Inc., New York, NY, USA, 2000.

[3] G. Sãlãgean, "Subclasses of univalent functions," in Complex Analysis-Fifth Romanian-Finnish Seminar, Part 1 (Bucharest, 1981), vol. 1013 of Lecture Notes in Mathematics, pp. 362-372, Springer, Berlin, Germany, 1983.

[4] S. Sivasubramanian, A. Mohammed, and M. Darus, "Certain subordination properties for subclasses of analytic functions involving complex order," Abstract and Applied Analysis, vol. 2011, Article ID 375897, 8 pages, 2011.

[5] M. K. Aouf, A. Shamandy, A. O. Mostafa, and E. A. Adwan, "Subordination results for certain classes of analytic functions defined by convolution with complex order," Bulletin of Mathematical Analysis and Applications, vol. 3, no. 1, pp. 61-68, 2011.

[6] G. Chunyi and S. Owa, "Certain class of analytic functions in the unit disk," Kyungpook Mathematical Journal, vol. 33, no. 1, pp. 13-23, 1993.

[7] M. P. Chen, "On the regular functions satisfying $\operatorname{Re}\{f(z) / z\}>$ $\alpha$, Bulletin of the Institute of Mathematics. Academia Sinica, vol. 3, no. 1, pp. 65-70, 1975.

[8] M. P. Chen, "On functions satisfying $\operatorname{Re}\{f(z) / z\}>\alpha$," Tamkang Journal of Mathematics, vol. 5, pp. 231-234, 1974.

[9] R. M. Goel, "On functions satisfying $\operatorname{Re}\{f(z) / z\}>\alpha$," Publicationes Mathematicae Debrecen, vol. 18, pp. 111-117, 1971.

[10] H. M. Srivastava and S. Owa, "Certain classes of analytic functions with varying arguments," Journal of Mathematical Analysis and Applications, vol. 136, no. 1, pp. 217-228, 1988.

[11] H. Silverman, "Univalent functions with varying arguments," Houston Journal of Mathematics, vol. 7, no. 2, pp. 283-287, 1981.

[12] M. K. Aouf, A. Shamandy, and A. A. Attiyia, "On certain classes of analytic functions with varying arguments," Chinese Journal of Mathematics, vol. 24, no. 2, pp. 135-143, 1996. 


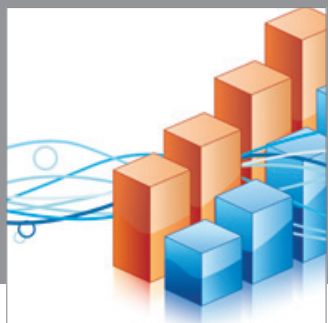

Advances in

Operations Research

mansans

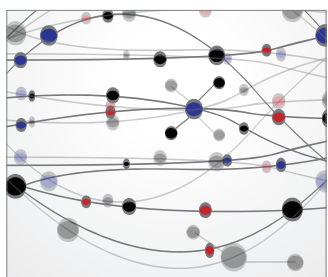

The Scientific World Journal
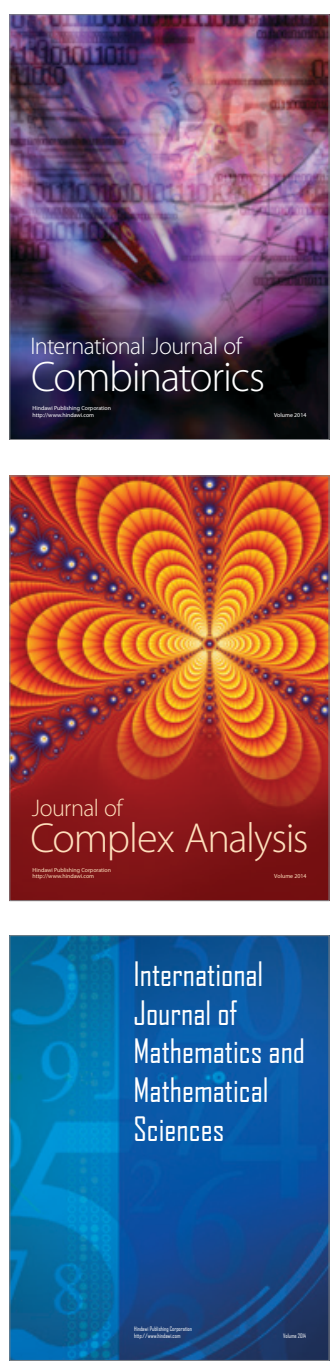
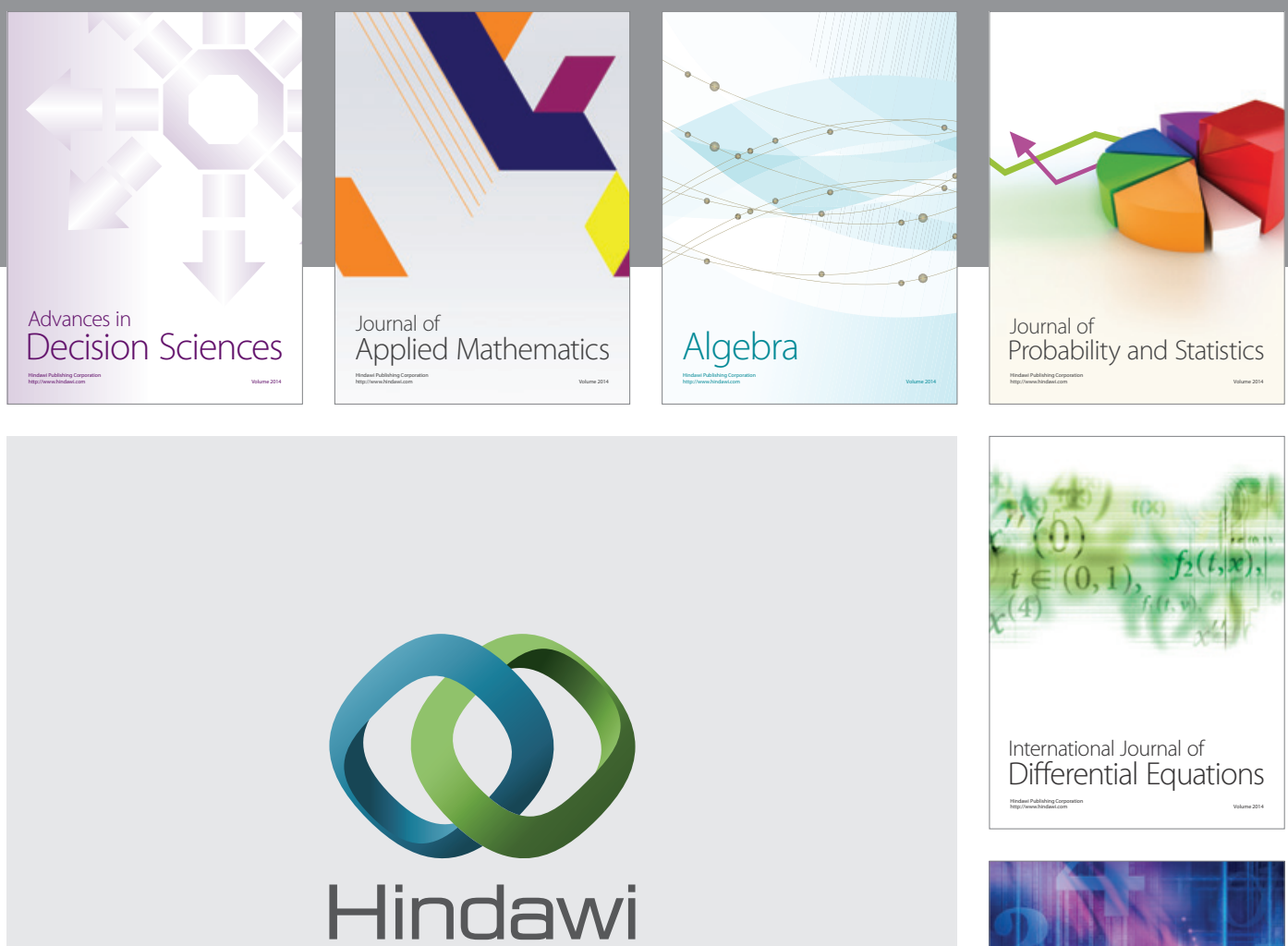

Submit your manuscripts at http://www.hindawi.com
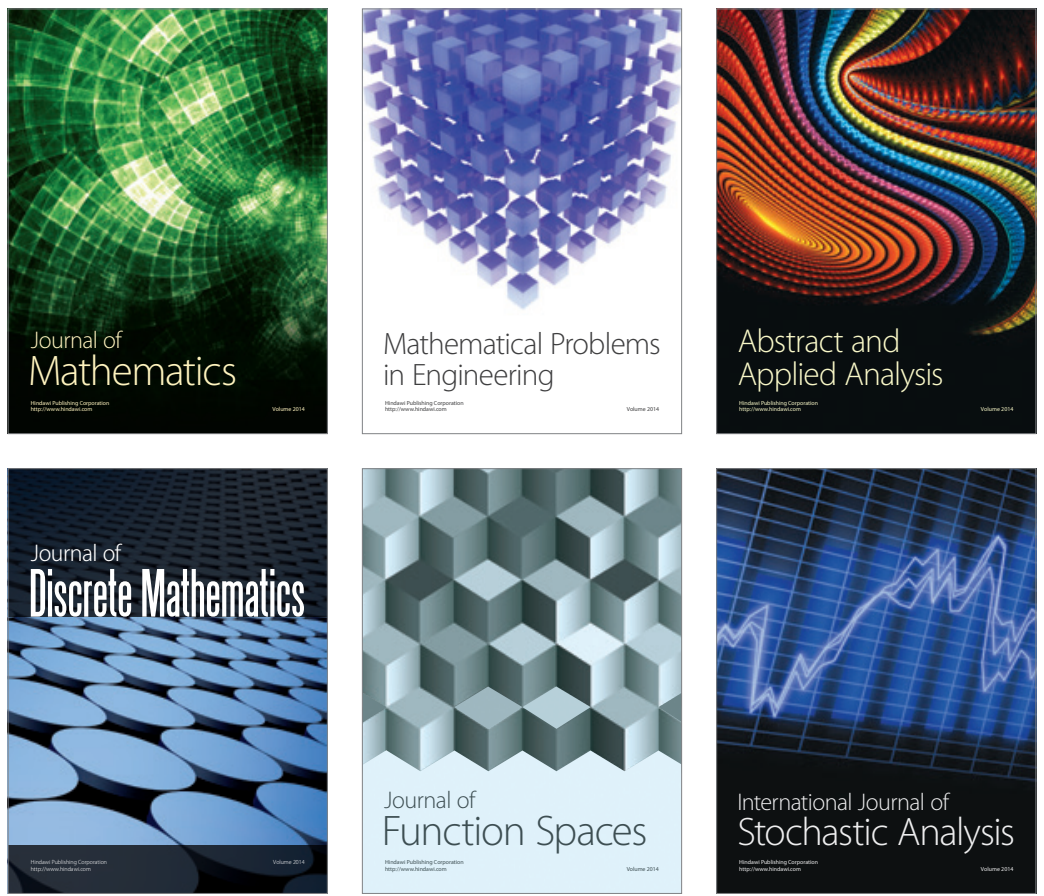

Journal of

Function Spaces

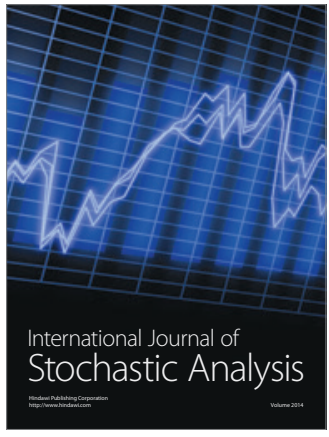

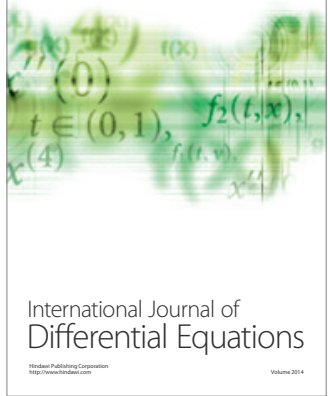
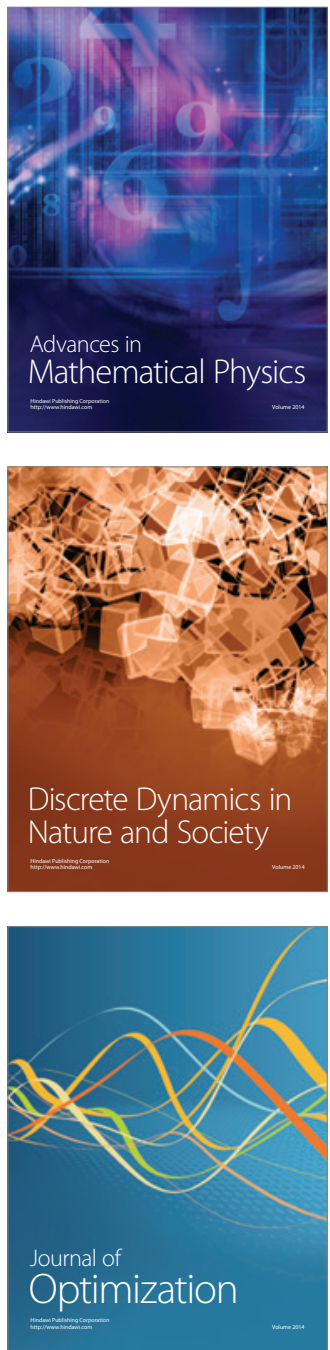